\title{
La crypte colique, site d'absorption d'eau et d'électrolytes: un nouveau concept en physiologie intestinale
}

En matière de transport d'eau et d'ions dans l'intestin grêle et le côlon, la séparation spatiale des fonctions d'absorption et de sécrétion entre, respectivement, les cellules épithéliales (entérocytes) de la surface muqueuse et celles des cryptes (parfois aussi dénommées glandes) situées en profondeur est un principe de physiologie intestinale admis depuis plus de 25 ans [1]. Il s'est fondé sur des preuves apparemment solides, mais plus indirectes que directes, telles que : (a) l'inhibition de l'effet sécrétoire (dans l'intestin grêle) de la toxine cholérique par des inhibiteurs de la synthèse protéique tels que le cycloheximide [2] qui lèse sélectivement les cryptes -siège de cellules en mitose - mais ne modifie pas l'absorption du glucose, ni le transfert de l'eau et du sodium dépendant du glucose -fonctions de l'entérocyte villositaire-; (b) inversement, la conservation de cet effet sécrétoire, parallèlement à la réduction de l'absorption du glucose, sous l'effet d'une forte hypertonie luminale [3] qui, quant à elle, lèse assez sélectivement les entérocytes villositaires ; (c) la non-réponse aux stimuli sécrétoires - dépendants de l'AMP cyclique - des rares intestins animaux dépourvus de cryptes, tels que celui d'un poisson, le carrelet [4, $5]$. Une approche plus directe fut assurée, dans un premier temps, par le recueil de succus entericus (fluide intraluminal «natif» de l'intestin à jeun) à l'aide de micropipettes disposées sur l'ostium des cryptes [6] puis, ultérieurement par l'insertion d'électrodes sur des entérocytes de surface et des entérocytes cryptiques de muqueuse colique [7]; ces expé- riences ont montré que le blocage par l'amiloride des canaux sodiques des membranes apicales diminuait bien la conductance membranaire des cellules de surface mais pas celle des cellules cryptiques et que, inversement, un sécrétagogue puissant comme la prostaglandine E2(PGE2), qui ouvre les canaux $\mathrm{Cl}^{-}$dans les cellules sécrétoires, augmentait la conductance apicale des cryptes sans modifier celle des cellules de surface, et provoquait une accumulation microscopique de gouttelettes liquidiennes sur l'orifice des cryptes.

Dix ans plus tard, plusieurs travaux ont suggéré que cette séparation spatiale fonctionnelle entre la surface muqueuse, qui ne ferait qu'absorber, et la profondeur - cryptique - qui ne ferait que sécréter, était une simplification probablement excessive de la réalité [8-10]. La possibilité d'une sécrétion des cellules de surface par ouverture de canaux $\mathrm{Cl}^{-}$fut soulevée par des études électrophysiologiques montrant une dépolarisation de surface dépendante du $\mathrm{Cl}^{-}$sous l'effet de sécrétagogues comme la PGE2 ou le dibutyryl-AMP cyclique $[8,9]$; inversement, celle d'une absorption hydroélectrolytique par les cryptes coliques fut suggérée par la visualisation du transfert de colorants fluorescents par celles-ci [10], sans que, néanmoins, la démonstration directe d'un flux hydro-ionique de la lumière cryptique vers le plasma ait été apportée. La lignée cellulaire T84, issue d'un cancer colique humain, a été largement utilisée comme modèle d'étude de la fonction cellulaire cryptique [11]; sensible à de nombreux agonistes et messagers intracellulaires à effet sécrétagogue, elle n'est, à l'état basal, le siège d'aucune absorption ni sécrétion hydroionique, et n'exprime donc probablement pas, du fait de sa filiation cancéreuse, les caractéristiques des cellules épithéliales cryptiques normales.

C'est pourquoi le récent travail de Singh et al. [12] (Université médicale de Yale, New Haven, USA) permet un important pas en avant. Il apporte la preuve expérimentale directe d'une absorption de fluide par les cryptes de côlon de rat, grâce à l'application à l'intestin de la technique de microperfusion jusque-là utilisée en physiologie rénale sur des segments isolés de néphron et, en physiologie digestive, sur des glandes gastriques. Après avoir très soigneusement isolé, par dissection microscopique manuelle, des cryptes coliques, ils ont vérifié l'intégrité anatomique, la polarité et la pureté de leurs préparations tissulaires, notamment l'absence de diverses cellules (myofibroblastes, cellules lymphoïdes, neurones entéraux) normalement situées dans la lamina propria au contact de la membrane basolatérale des entérocytes cryptiques, et libérant des neuromédiateurs qui modulent de façon paracrine la fonction cryptique; c'est notamment le cas des myofibroblastes péricryptiques, sécréteurs de prostaglandines. Ils ont ensuite microponctionné le versant borgne de la crypte afin de perfuser sa lumière et recueilli l'effluent par cannulation du versant ouvert opposé. Ainsi ont-ils pu démontrer une absorption d'eau par la crypte, dépendante du sodium; cette dernière répondait aussi à l'effet de divers sécrétagogues, comme le dibutyryl-AMP cyclique ou le VIP (vasoacti- 
ve intestinal peptide, qui est un puissant stimulant de l'adénylyl cyclase intestinale) ou, comme l'acétylcholine, neurotransmetteur stimulant la sécrétion active de chlorure dans le grêle et le côlon par augmentation du calcium intracellulaire.

L'absorption colique du sodium est électriquement neutre et compatible avec un échange $\mathrm{Na}^{+}-\mathrm{H}^{+}$: l'absorption liquidienne, dépendante du sodium, par les cryptes coliques, démontrée par Singh et al. [12], pourraît être, ainsi, à mettre sur le compte du nouveau système - dépendant du $\mathrm{Cl}^{-}-$ d'échange $\mathrm{Na}^{+}-\mathrm{H}^{+}$récemment mis en évidence dans la membrane apicale d'entérocytes cryptiques de rat [13]. Il est également probable que, dans l'intestin entier, les cryptes voient leur fonction modulée par des médiateurs paracrines libérés par les cellules péricryptiques - myofibroblastes, cellules lymphoïdes, neurones entéraux - et n'y soient pas en situation d'absorption mais de sécrétion nette. Même si elle est normalement masquée, la fonction d'absorption hydroionique par les cryptes intestinales pourrait avoir d'importantes implications tant physiologiques que pathologiques. En physiologie, elle pourrait être l'élément régulateur principal de la fluidité du contenu colique intraluminal ; au niveau de l'intestin grêle - qui n'a pas été étudié par Singh et al. [12] -, son existence et sa signification restent, en revanche, à prouver. En pathologie, les lésions histologiques des cryptes, qui sont régulièrement observées au cours des maladies inflammatoires chroniques de l'intestin -maladie de Crohn, rectocolite hémorragique, colites inclassées-, pourraient avoir, en complément des lésions de surface, un important retentissement sur les flux hydroioniques, la réduction voire la suppression de l'absorption cryptique s'ajoutant à celle des entérocytes de surface pour augmenter les pertes hydro-ioniques nettes dans la lumière colique. La technique de microponction ajoute donc un outil précieux à l'arsenal de la recherche en physiologie digestive.
1. Hendrix TR, Bayless TM. Digestion : intestinal secretion. Annu Rev Physiol 1970 ; 32 : 139-64.

2. Serebro HA, Iber FL, Yardley JH, Hendrix TR. Inhibition of cholera toxin action in the rabbit by cycloheximide. Gastroenterology 1969 ; 56 : 506-11. 3. McGonagle TJ, Serebro HA, Iber FL, Bayless TM, Hendrix TR. Time of onset of action of cholera toxin in dog and rabbit. Gastroenterology 1969 ; $57: 5-8$.

4. Field M. Regulation of small intestinal ion transport by cyclic nucleotides and calcium. In: Field M, Fordtran JS, Schultz SG eds. Secretory diarrhea. Bethesda American Physiological Society, 1980: $21-30$

5. Powell DH. Dogma destroyed : colonic crypts absorb. J Clin Invest 1995 ; 96 : 2102-3.

6. Nasset ES, Ju JS. Micropipet collection of succus entericus at crypt ostia of guinea pig jejunum. Digestion 1973 ; 9 : 205-11.

7. Welch MJ, Smith PL, Fromm M, Frizzell RA. Crypts are the site of intestinal fluid and electrolyte secretion. Science $1982 ; 218: 1219-21$.

8. Stewart CP, Turnberg LA. A microelectrode study of responses to secretagogues by epithelial cells on villus and crypt of rat small intestine. $\mathrm{Am}$ J Physiol 1989 ; 257 : G334-43.

9. Kockerling A, Fromm M. Origin of cAMP-dependent Cl-secretion from both crypts and surface epithelia of rat intestine. Am J Physiol 1993 ; 364 : C1294-301.

10. Naftalin RJ, Peydley KC. Video enhanced imaging of the fluorescent Na-probe SBFI indicates that colonic crypts absorb fluid by generating a hypertonic interstitial fluid. FEBS Lett 1990; 260 : 187-94.

11. Dharmsathaphorn K, McRoberts JA, Mandel KG, Tisdale LD, Masui H. A human colonic tumor cell that maintains vectorial electrolyte transport. Am J Physiol 1984 ; 246 : G204-8.

12. Singh KS, Binder HJ, Boron WF, Geibel JP. Fluid absorption in isolated perfused colonic crypts. J Clin Invest $1995 ; 96$ : 2373-9.

13. Rajendran VM, Geibel JP, Binder HJ. Chloride dependent Na-H exchange : a novel mechanism of $\mathrm{Na}^{+}$transport in colonic crypts. I Biol Chem 1995 ; 270 : 11051-4. 